Bull. Austral. Math. Soc.

$05 \mathrm{~B} 15,05 \mathrm{~B} 20,62 \mathrm{\kappa} 05$

VOL. 64 (2001) [495-504]

\title{
SHORT AMICABLE SETS AND KHARAGHANI TYPE ORTHOGONAL DESIGNS
}

Christos Koukouvinos and Jennifer Seberry

\section{Dedicated to Professor George Szekeres}

\begin{abstract}
Short amicable sets were introduced recently and have many applications. The construction of short amicable sets has lead to the construction of many orthogonal designs, weighing matrices and Hadamard matrices. In this paper we give some constructions for short amicable sets as well as some multiplication theorems. We also present a table of the short amicable sets known to exist and we construct some infinite families of short amicable sets and orthogonal designs.
\end{abstract}

\section{INTRODUCTION}

An orthogonal design of order $n$ and type $\left(s_{1}, s_{2}, \ldots, s_{u}\right)$ denoted $O D\left(n ; s_{1}, s_{2}, \ldots, s_{u}\right)$ in the variables $x_{1}, x_{2}, \ldots, x_{u}$, is a matrix $A$ of order $n$ with entries in the set $\left\{0, \pm x_{1}, \pm x_{2}\right.$, $\left.\ldots, \pm x_{u}\right\}$ satisfying

$$
A A^{T}=\sum_{i=1}^{u}\left(s_{i} x_{i}^{2}\right) I_{n},
$$

where $I_{n}$ is the identity matrix of order $n$. Let $B_{i}, i=1,2,3,4$ be circulant matrices of order $n$ with entries in $\left\{0, \pm x_{1}, \pm x_{2}, \ldots, \pm x_{u}\right\}$ satisfying

$$
\sum_{i=1}^{4} B_{i} B_{i}^{T}=\sum_{i=1}^{u}\left(s_{i} x_{i}^{2}\right) I_{n}
$$

Then the Goethals-Seidel array

$$
G=\left(\begin{array}{cccc}
B_{1} & B_{2} R & B_{3} R & B_{4} R \\
-B_{2} R & B_{1} & B_{4}^{T} R & -B_{3}^{T} R \\
-B_{3} R & -B_{4}^{T} R & B_{1} & B_{2}^{T} R \\
-B_{4} R & B_{3}^{T} R & -B_{2}^{T} R & B_{1}
\end{array}\right)
$$

where $R$ is the back-diagonal identity matrix, is an $O D\left(4 n ; s_{1}, s_{2}, \ldots, s_{u}\right)$. See [2, p.107] for details.

Received 24th May, 2001

Copyright Clearance Centre, Inc. Serial-fee code: 0004-9727/01 \$A2.00+0.00. 
A pair of matrices $A, B$ is said to be amicable (anti-amicable) if $A B^{T}-B A^{T}=0$ $\left(A B^{T}+B A^{T}=0\right)$. To be consistent in the notation of this paper we shall also denote these as $2-S A S\left(n ; s_{1}, s_{2} ; G\right)$, where the group $G$ is described below. Following [4] a set $\left\{A_{1}, A_{2}, \ldots, A_{2 n}\right\}$ of square real matrices is said to be amicable if

$$
\sum_{i=1}^{n}\left(A_{\sigma(2 i-1)} A_{\sigma(2 i)}^{T}-A_{\sigma(2 i)} A_{\sigma(2 i-1)}^{T}\right)=0
$$

for some permutation $\sigma$ of the set $\{1,2, \ldots, 2 n\}$. For simplicity, we shall always take $\sigma(i)=i$ unless otherwise specified. So

$$
\sum_{i=1}^{n}\left(A_{2 i-1} A_{2 i}^{T}-A_{2 i} A_{2 i-1}^{T}\right)=0
$$

Clearly a set of mutually amicable matrices is amicable, but the converse is not true in general. Throughout the paper $R_{k}$ denotes the back diagonal identity matrix of order $k$.

A set of matrices $\left\{B_{1}, B_{2}, \ldots, B_{n}\right\}$ of order $m$ with entries in $\left\{0, \pm x_{1}, \pm x_{2}, \ldots, \pm x_{u}\right\}$ is said to satisfy an additive property of type $\left(s_{1}, s_{2}, \ldots, s_{u}\right)$ if

$$
\sum_{i=1}^{n} B_{i} B_{i}^{T}=\sum_{i=1}^{u}\left(s_{i} x_{i}^{2}\right) I_{m}
$$

Let $\left\{A_{i}\right\}_{i=1}^{8}$ be an amicable set of circulant matrices (or group developed or type 1) of type $\left(s_{1}, s_{2}, \ldots, s_{u}\right)$ and order $t$. We denote these by $8-A S\left(t ; s_{1}, s_{2}, s_{3}, s_{4}, s_{5}, s_{6}, s_{7}, s_{8} ; Z_{t}\right)$ (or $8-A S\left(t ; s_{1}, s_{2}, s_{3}, s_{4}, s_{5}, s_{6}, s_{7}, s_{8} ; G\right.$ ) for group developed or type 1 ). In all cases, the group $G$ of the matrix is such that the extension by Seberry and Whiteman [7] of the group from circulant to type 1 allows the same extension to $R$. Then the Kharaghani array $[4]$

$$
H=\left(\begin{array}{cccccccc}
A_{1} & A_{2} & A_{4} R_{n} & A_{3} R_{n} & A_{6} R_{n} & A_{5} R_{n} & A_{8} R_{n} & A_{7} R_{n} \\
-A_{2} & A_{1} & A_{3} R_{n} & -A_{4} R_{n} & A_{5} R_{n} & -A_{6} R_{n} & A_{7} R_{n} & -A_{8} R_{n} \\
-A_{4} R_{n} & -A_{3} R_{n} & A_{1} & A_{2} & -A_{8}^{T} R_{n} & A_{7}^{T} R_{n} & A_{6}^{T} R_{n} & -A_{5}^{T} R_{n} \\
-A_{3} R_{n} & A_{4} R_{n} & -A_{2} & A_{1} & A_{7}^{T} R_{n} & A_{8}^{T} R_{n} & -A_{5}^{T} R_{n} & -A_{6}^{T} R_{n} \\
-A_{6} R_{n} & -A_{5} R_{n} & A_{8}^{T} R_{n} & -A_{7}^{T} R_{n} & A_{1} & A_{2} & -A_{4}^{T} R_{n} & A_{3}^{T} R_{n} \\
-A_{5} R_{n} & A_{6} R_{n} & -A_{7}^{T} R_{n} & -A_{8}^{T} R_{n} & -A_{2} & A_{1} & A_{3}^{T} R_{n} & A_{4}^{T} R_{n} \\
-A_{8} R_{n} & -A_{7} R_{n} & -A_{6}^{T} R_{n} & A_{5}^{T} R_{n} & A_{4}^{T} R_{n} & -A_{3}^{T} R_{n} & A_{1} & A_{2} \\
-A_{7} R_{n} & A_{8} R_{n} & A_{5}^{T} R_{n} & A_{6}^{T} R_{n} & -A_{3}^{T} R_{n} & -A_{4}^{T} R_{n} & -A_{2} & A_{1}
\end{array}\right)
$$

is an $O D\left(8 t ; s_{1}, s_{2}, \ldots, s_{u}\right)$.

The Kharaghani array has been used in a number of papers $[1,3,4]$ to obtain infinitely many families of orthogonal designs. Research has yet to be initiated to explore the algebraic restrictions imposed on amicable set by the required constraints. 
Short amicable sets were defined in $[1]$ as a set of matrices $\left\{A_{i}\right\}_{i=1}^{4}$ of order $m$ and type $\left(u_{1}, u_{2}, u_{3}, u_{4}\right)$, abbreviated as $4-S A S\left(m ; u_{1}, u_{2}, u_{3}, u_{4} ; G\right)$, if (2) and (3) are satisfied for $n=4$ and $u \leqslant 4.4-S A S\left(m ; u_{1}, u_{2}, u_{3}, u_{4} ; G\right)$ can be used in either the Goethals-Seidel array or the short Kharaghani array

$$
\left[\begin{array}{cccc}
A & B & C R & D R \\
-B & A & D R & -C R \\
-C R & -D R & A & B \\
-D R & C R & -B & A
\end{array}\right]
$$

to form an $O D\left(4 m ; u_{1}, u_{2}, u_{3}, u_{4}\right)$. In all cases, the group $G$ of the matrices in the amicable set is such that the extension by Seberry and Whiteman [7] of the group from circulant to type 1 allows the same extension to $R$.

In general a set of $2 n$ matrices of order $m$ and type $\left(s_{1}, s_{2}, \ldots, s_{u}\right)$ that satisfy equations (2) and (3) will be denoted as $2 n-S A S\left(m ; s_{1}, s_{2}, \ldots, s_{u} ; G\right)$. Moreover if these matrices are circulant they will be denoted as $2 n-S C A S\left(m ; s_{1}, s_{2}, \ldots, s_{u} ; Z_{m}\right)$.

In [1] where all this was first defined was mentioned that:

REMARK 1.

1. If there exists a $2-S A S\left(n ; s_{1}, s_{2} ; G\right)$ and a $2-S A S\left(n ; s_{3}, s_{4} ; G\right)$ then there exists a $4-S A S\left(n ; s_{1}, s_{2}, s_{3}, s_{4} ; G\right)$.

2. If there exists a $2-S A S\left(n ; s_{1}, s_{2} ; G\right), 2-S A S\left(n ; s_{3}, s_{4} ; G\right), 2-S A S\left(n ; s_{5}, s_{6} ; G\right)$ and a $2-S A S\left(n ; s_{7}, s_{8} ; G\right)$ there exists an $8-A S\left(n ; s_{1}, s_{2}, s_{3}, s_{4}, s_{5}, s_{6}, s_{7}, s_{8} ; G\right)$.

3. If there exists a $4-S A S\left(n ; s_{1}, s_{2}, s_{3}, s_{4} ; G\right)$ and a $4-S A S\left(n ; s_{5}, s_{6}, s_{7}, s_{8} ; G\right)$ there exists an $8-A S\left(n ; s_{1}, s_{2}, s_{3}, s_{4}, s_{5}, s_{6}, s_{7}, s_{8} ; G\right)$.

Thus we can obtain many classes of $4-S A S\left(n ; s_{1}, s_{2}, s_{3}, s_{4} ; G\right)$ combining together two pairs of the given $2-S A S\left(n ; s_{1}, s_{2} ; G\right)$ and $2-S A S\left(n ; s_{3}, s_{4} ; G\right)$. Moreover, in Table 2 , we give some $4-S A S\left(m ; u_{1}, u_{2}, u_{3}, u_{4} ; Z_{m}\right)$ that can not be constructed by this method.

Generally, unless we have other information regarding the structure, we are unable to ensure that the matrix $R$ with the desired properties for the Kharaghani, Goethals-Seidel or short Kharaghani arrays exists unless the amicable sets have been group generated (circulant or type 1) or constructed from blocks of these kinds. Thus if we have the required matrix $R_{i}$ for the group $G_{i}, i=1,2$ then $R_{G}=R_{1} \times R_{2}$ will be the required matrix for $G=G_{1} \times G_{2}$, (see [7]).

Let $A_{1}$ and $A_{2}$ be matrices of order $m$. We define $\operatorname{circ}\left(A_{1}, A_{2}\right)=\left[\begin{array}{cc}A_{1} & A_{2} \\ A_{2} & A_{1}\end{array}\right]$. Amicable sets made from $2 n$ such block circulant matrices will be called block amicable sets, short block amicable sets or 2-short block amicable sets, $2 n-S B A S\left(2 m ; s_{1}, s_{2}, \ldots, s_{u} ; G\right)$, $n=1,2,4$, where, using $R_{t}$ for the back-diagonal matrix of order $t, G=Z_{2} \times Z_{m}$ and $R_{G}=R_{2} \times R_{m}$. Here, if $A_{1}$ and $A_{2}$ are circulant, then we use the backdiagonal matrix of the same order for $R$ ensuring $A_{i}\left(A_{j} R\right)^{T}=A_{j} R A_{i}^{T}$. The required $R_{G}=R_{2} \times R_{m}$. 
A $(1,-1)$ matrix of order $n$ is called a Hadamard matrix if $H H^{T}=H^{T} H=n I_{n}$, where $H^{T}$ is the transpose of $H$ and $I_{n}$ is the identity matrix of order $\mathbf{n}$. A $(1,-1)$ matrix $A$ of order $n$ is said to be of skew type if $A-I_{n}$ is skew-symmetric.

A matrix $W=\operatorname{circ}\left(w_{1}, \ldots, w_{n}\right), w_{i} \in\{0, \pm 1\}$ which satisfies $W W^{T}=k I_{n}$ is called a circulant weighing matrix of order $n$ and weight $k$ or $C W(n, k)$.

We denote the product $Z_{p} \times Z_{p} \times \cdots \times Z_{p}(r$ times $)$ by $E A\left(p^{r}\right)$ the Elementary Abelian group. Moreover $-a$ is denoted by $\bar{a}$.

Throughought this paper we use the symbol $0_{m}$ to denote the sequence of length $m$ with all elements zero and the symbol $O_{t}$ to denote the $t \times t$ matrix with all entries zero.

For the undefined terms we refer the reader to the book by Geramita and Seberry [2].

Suppose $C=\operatorname{circ}\left(c_{0}, c_{1}, \ldots, c_{n-1}\right)$ is a circulant matrix of order $n$. Let

$$
T_{n}=\left[\begin{array}{cccccc}
0 & 1 & 0 & 0 & \ldots & 0 \\
0 & 0 & 1 & 0 & \ldots & 0 \\
\vdots & & & & & \vdots \\
0 & 0 & 0 & 0 & \ldots & 1 \\
1 & 0 & 0 & 0 & \ldots & 0
\end{array}\right]
$$

of order $n$, be the shift matrix. Then we can write $C=c_{0} I+c_{1} T_{n}+\ldots+c_{n-1} T_{n}^{n-1}$. Note that $T_{n}^{n}=I$ the identity matrix of order $n$. We say the Hall polynomial of $C$ is $\sum_{i=0}^{n-1} c_{i} x^{i}$. The Hall polynomial of $C^{T}$ is $\sum_{i=0}^{n-1} c_{i} x^{n-i}$.

Given a set of $\ell$ sequences $A_{j}=\left\{a_{j 1}, a_{j 2}, \ldots, a_{j n}\right\}, j=1, \ldots, \ell$, of length $n$ the non-periodic autocorrelation function, denoted $N P A F, N_{A}(s)$ is defined as

$$
N_{A}(s)=\sum_{j=1}^{\ell} \sum_{i=1}^{n-s} a_{j i} a_{j, i+s}, \quad s=0,1, \ldots, n-1
$$

If $A_{j}(z)=a_{j 1}+a_{j 2} z+\ldots+a_{j n} z^{n-1}$ is the associated polynomial of the sequence $A_{j}$, then

$$
A(z) A\left(z^{-1}\right)=\sum_{j=1}^{\ell} \sum_{i=1}^{n} \sum_{k=1}^{n} a_{j i} a_{j k} z^{i-k}=N_{A}(0)+\sum_{j=1}^{\ell} \sum_{s=1}^{n-1} N_{A}(s)\left(z^{s}+z^{-s}\right), z \neq 0 .
$$

Given $A_{\ell}$, as above, of length $n$ the periodic autocorrelation function, denoted $P A F$, $P_{A}(s)$ is defined, reducing $i+s$ modulo $n$, as

$$
P_{A}(s)=\sum_{j=1}^{\ell} \sum_{i=1}^{n} a_{j i} a_{j, i+s}, \quad s=0,1, \ldots, n-1 .
$$

We note NPAF sequences imply PAF sequences exist, the NPAF sequences being padded at the end with sufficient zeros to make longer lengths. Hence NPAF sequences 
can give more general results. If two NPAF sequences have differing lengths then sufficient zeros are added to the end of each to make all the sequences the same length. In all cases NPAF and PAF sequences can be used to make circulant matrices satisfying the additive property (see $[3,4]$ ); if NPAF sequences of lengths $n_{1}$ and $n_{2}$ are used, then by padding, circulant matrices for all orders $n \geqslant \max \left(n_{1}, n_{2}\right)$ will exist; if PAF sequences of lengths $n$ are used, then circulant matrices of order $n$ exist.

\section{Constructions}

THEOREM 1. Write $0_{s}$ for the sequence of $s$ zeros, and let $a, b, c$ and $d$ be commuting variables. Use the matrices $A_{1}, A_{2}, A_{3}$ and $A_{4}$ given by

$$
\begin{array}{ll}
A_{1}=\operatorname{circ}\left(0_{s} b a \bar{b} 0_{s}\right), & A_{2}=\operatorname{circ}\left(0_{s} c 0 c 0_{s}\right), \\
A_{3}=\operatorname{circ}\left(0_{s} \bar{c} \bar{d} c 0_{s}\right), & A_{4}=\operatorname{circ}\left(0_{s} b 0 b 0_{s}\right),
\end{array}
$$

can be used in the Goethals-Seidel array to obtain an $O D(8 s+12 ; 1,1,4,4)$.

Proof: Observe that

$$
A_{1} A_{1}^{T}+A_{2} A_{2}^{T}+A_{3} A_{3}^{T}+A_{4} A_{4}^{T}=\left(a^{2}+d^{2}+4 b^{2}+4 d^{2}\right) I_{n}
$$

and

$$
A_{1} A_{1}^{T}-A_{2} A_{2}^{T}+A_{3} A_{3}^{T}-A_{4} A_{4}^{T}=0 .
$$

Thus $A_{2}, A_{2}, A_{3}, A_{4}$ are a short amicable set and satisfy the additive property (2) so they can be used in the Goethals-Seidel array to obtain an $O D(8 s+12 ; 1,1,4,4)$.

The Melding Construction. Suppose the matrices $A_{1}, A_{2}, A_{3}$ and $A_{4}$ are are short amicable sets, on the set of commuting variables $\left\{0, \pm x_{1}, \pm x_{2}, \cdots, \pm x_{u}\right\}$ or from $\{0, \pm 1\}$, and satisfy the additive property

$$
\sum_{i=1}^{4}\left(A_{i} A_{i}^{T}\right)=\sum_{j=1}^{u} p_{j} x_{j}^{2} I_{n},
$$

and the matrices $A_{5}, A_{6}, A_{7}$ and $A_{8}$ are also short amicable sets, on the set of commuting variables $\left\{0, \pm y_{1}, \pm y_{2}, \cdots, \pm y_{v}\right\}$ or from $\{0, \pm 1\}$, and satisfy the additive property

$$
\sum_{i=5}^{8}\left(A_{i} A_{i}^{T}\right)=\sum_{j=1}^{v} q_{j} y_{j}^{2} I_{n} .
$$

Then the eight matrices will form an amicable set so we can use the two together in the Kharaghani array to obtain an $O D\left(8 n ; p_{1}, p_{2}, \cdots, p_{u}, q_{1}, q_{2}, \cdots, q_{v}\right)$.

Using Table 2, Remark 1 and the following Melding Construction we obtain many 4-short amicable sets and 8-amicable sets. 


\begin{tabular}{||c|c|c||c|c|c||c|c|c||c|c|c||}
\hline order & type & group & order & type & group & order & type & group & order & type & group \\
\hline$n$ & 1,1 & $Z_{n}$ & $6 n$ & 4,4 & $Z_{6 n}$ & $10 n$ & 4,4 & $Z_{10 n}$ & $14 n$ & 8,8 & $Z_{14 n}$ \\
$2 n$ & 2,2 & $Z_{2 n}$ & $6 n$ & 5,5 & $Z_{6 n}$ & $10 n$ & 9,9 & $Z_{10 n}$ & $14 n$ & 10,10 & $Z_{14 n}$ \\
$4 n$ & 1,4 & $Z_{4 n}$ & $7 n$ & 4,4 & $Z_{7 n}$ & $12 n$ & 8,8 & $Z_{12 n}$ & $14 n$ & 13,13 & $Z_{14 n}$ \\
$4 n$ & 4,4 & $Z_{4 n}$ & $8 n$ & 8,8 & $Z_{8 n}$ & $13 n$ & 9,9 & $Z_{13 n}$ & & & \\
\hline
\end{tabular}

Table 1: Order and type for small 2 -short amicable sets for all $n \geqslant 1$.

\begin{tabular}{|c|c|c|c|}
\hline Type & $\begin{array}{l}A_{1} \\
A_{2}\end{array}$ & $\begin{array}{l}A_{3} \\
A_{4}\end{array}$ & ZERO \\
\hline$(1,1,1,1)$ & $\begin{array}{l}\mathrm{a} \\
\mathrm{c}\end{array}$ & $\begin{array}{l}\mathrm{b} \\
\mathrm{d}\end{array}$ & $\begin{array}{c}\text { NPAF } \\
n\end{array}$ \\
\hline$(1,1,1,4)$ & $\begin{array}{c}0-d \text { a d } \\
0 \mathrm{~d} 0 \mathrm{~d}\end{array}$ & $\begin{array}{llll}0 & \mathrm{~b} & 0 & 0 \\
0 \mathrm{c} & 0 & 0\end{array}$ & $\begin{array}{c}\text { NPAF } \\
4 n\end{array}$ \\
\hline$(1,1,2,2)$ & $\begin{array}{ll}\text { a } & 0 \\
\text { b } & 0\end{array}$ & $\begin{array}{c}c d \\
c-d\end{array}$ & $\begin{array}{c}\text { NPAF } \\
2 n\end{array}$ \\
\hline$(1,1,2,8)$ & $\begin{array}{l}0-c \text { a c } \\
0 \text { c b c }\end{array}$ & $\begin{array}{l}0-c \quad b-c \\
0-c d c\end{array}$ & $\begin{array}{c}\text { NPAF } \\
4 n\end{array}$ \\
\hline$(1,1,4,4)$ & $\begin{array}{l}a b-a \\
c 0 c\end{array}$ & $\begin{array}{l}a 0 a \\
c d-c\end{array}$ & $\begin{array}{c}\text { NPAF } \\
3 n\end{array}$ \\
\hline$(1,1,5)$ & $\begin{array}{lll}-\mathrm{a} & \mathrm{a} & \mathrm{a} \\
\mathrm{c} & 0 & 0\end{array}$ & $\begin{array}{l}\mathrm{a} 0 \mathrm{a} \\
0 \mathrm{~b} 0\end{array}$ & $\begin{array}{c}\text { NPAF } \\
4 n\end{array}$ \\
\hline$(1,1,5,5)$ & 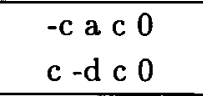 & $\begin{array}{c}-\mathrm{d} b \mathrm{~d} 0 \\
\mathrm{~d} \text { c d } 0\end{array}$ & $\begin{array}{c}\text { NPAF } \\
4 n\end{array}$ \\
\hline$(1,1,8,8)$ & $\begin{array}{c}0-c-d a d c \\
0 c d 0 d c\end{array}$ & $\begin{array}{l}0 \mathrm{c}-\mathrm{d} 0-\mathrm{d} c \\
0-\mathrm{c} d \mathrm{~b}-\mathrm{d} \mathrm{c}\end{array}$ & $\begin{array}{c}\text { NPAF } \\
6 n \\
\end{array}$ \\
\hline$(1,2,2,4)$ & $\begin{array}{l}0-\mathrm{d} a \mathrm{~d} \\
0 \mathrm{~d} 0 \mathrm{~d}\end{array}$ & $\begin{array}{c}\text { c } 0 \text { b } 0 \\
\text { c } 0-b \quad-b\end{array}$ & $\begin{array}{c}\text { NPAF } \\
4 n\end{array}$ \\
\hline$(1,4,4,4)$ & 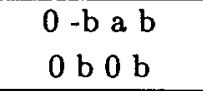 & $\begin{array}{l}d c-d c \\
-c d c d\end{array}$ & $\begin{array}{c}\text { NPAF } \\
4 n\end{array}$ \\
\hline$(2,2,2,2)$ & $\begin{array}{l}\mathrm{a} b \\
\mathrm{c} \mathrm{d}\end{array}$ & $\begin{array}{l}a-b \\
c-d\end{array}$ & $\begin{array}{c}\text { NPAF } \\
2 n\end{array}$ \\
\hline$(2,2,4,4)$ & 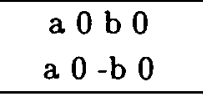 & $\begin{array}{l}d c-d c \\
-c d c d \\
\end{array}$ & $\begin{array}{c}\text { NPAF } \\
4 n\end{array}$ \\
\hline$(2,2,5,5)$ & 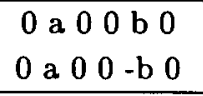 & $\begin{array}{l}c-d 0-d c d \\
d ~ c 0 c d-c\end{array}$ & $\begin{array}{c}\text { NPAF } \\
6 n \\
\end{array}$ \\
\hline$(2,2,8,8)$ & $\begin{array}{c}-d \text { c a c d } 0 \\
-d-c \text { a }-c \text { d } 0\end{array}$ & $\begin{array}{c}d-c b c d 0 \\
-d-c b c-d 0\end{array}$ & $\begin{array}{c}\text { NPAF } \\
6 n\end{array}$ \\
\hline
\end{tabular}

Table 2: Short amicable sets. 


\begin{tabular}{|c|c|c|c|}
\hline Type & $\begin{array}{l}A_{1} \\
A_{2}\end{array}$ & $\begin{array}{l}A_{3} \\
A_{4}\end{array}$ & ZERO \\
\hline$(3,3)$ & $\begin{array}{ll}\mathrm{a} & \mathrm{b} \\
\mathrm{a} & 0\end{array}$ & $\begin{array}{l}\mathrm{b}-\mathrm{a} \\
\mathrm{b} 0\end{array}$ & $\begin{array}{c}\text { NPAF } \\
2 n\end{array}$ \\
\hline$(4,4,4,4)$ & $\begin{array}{l}a \mathrm{a} b-\mathrm{b} \\
\mathrm{d} d-\mathrm{c} c\end{array}$ & $\begin{array}{l}\text { b b-a a } \\
\text { c c d-d }\end{array}$ & $\begin{array}{c}\text { NPAF } \\
4 n \\
\end{array}$ \\
\hline$(4,4,8,8)$ & $\begin{array}{l}d a-c c a-d \\
-d-b \quad c \quad c b-d\end{array}$ & $\begin{array}{c}d b c-c b-d \\
d-a c c a d\end{array}$ & $\begin{array}{c}\text { NPAF } \\
6 n\end{array}$ \\
\hline$(5,5)$ & $\begin{array}{l}a-a-a \\
b-b-b\end{array}$ & $\begin{array}{l}\text { a } 0 \mathrm{a} \\
\text { b } 0 \mathrm{~b}\end{array}$ & $\begin{array}{c}\text { NPAF } \\
3 n\end{array}$ \\
\hline$(5,5,5,5)$ & $\begin{array}{l}-a b a 0 a b \\
b a-b 0-b a\end{array}$ & $\begin{array}{c}-c d c 0 c d \\
d c-d 0-d c\end{array}$ & $\begin{array}{c}\text { NPAF } \\
6 n\end{array}$ \\
\hline$(6,6)$ & $\begin{array}{l}a-b a \\
b a b\end{array}$ & $\begin{array}{l}a-a-a \\
b \quad b-b\end{array}$ & $\begin{array}{c}\text { NPAF } \\
3 n \\
\end{array}$ \\
\hline$(6,6,12)$ & $\begin{array}{r}c \text { a c b-c a } \\
-c \text { b-c-a c b }\end{array}$ & $\begin{array}{r}c \text { a c-a c-a } \\
-c \text { b c-b-c-b }\end{array}$ & $\begin{array}{c}\text { NPAF } \\
6 n \\
\end{array}$ \\
\hline$(8,8)$ & $\begin{array}{l}a \text { a } a-a \\
b \text { b b-b }\end{array}$ & $\begin{array}{l}\text { b b-b b } \\
a \text { a-a a }\end{array}$ & $\begin{array}{c}\text { NPAF } \\
4 n\end{array}$ \\
\hline$(8,8,8,8)$ & $\begin{array}{l}a \text { a } a-a \quad b \quad b-b \quad b \\
c \text { c c-c d d-d d }\end{array}$ & $\begin{array}{l}b \text { b b-b a a-a a } \\
d d d-d c \text { c-c c }\end{array}$ & $\begin{array}{c}\text { NPAF } \\
8 n\end{array}$ \\
\hline$(10,10,10,10)$ & disjoint & from Golay & $\begin{array}{l}\text { NPAF } \\
n \geqslant 10\end{array}$ \\
\hline$(13,13)$ & 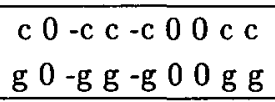 & 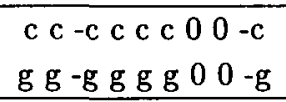 & $\begin{array}{c}\text { NPAF } \\
9 n \\
\end{array}$ \\
\hline$(13,13,13,13)$ & \multicolumn{2}{|c|}{$\begin{array}{c}\text { from disjoint sequences } \\
\text { of length } 18 \text { and weight } 13\end{array}$} & $\begin{array}{l}\text { NPAF } \\
n \geqslant 18\end{array}$ \\
\hline$(16,16,16,16)$ & disjoint & from Golay & $\begin{array}{l}\text { NPAF } \\
n \geqslant 16\end{array}$ \\
\hline$(17,17,17,17)$ & \multicolumn{2}{|c|}{$\begin{array}{l}\text { from disjoint sequences } \\
\text { of length } 26 \text { and weight } 17\end{array}$} & $\begin{array}{l}\text { NPAF } \\
n \geqslant 26\end{array}$ \\
\hline$(20,20,20,20)$ & disjoint & from Golay & $\begin{array}{l}\text { NPAF } \\
n \geqslant 20\end{array}$ \\
\hline$(25,25,25,25)$ & \multicolumn{2}{|c|}{$\begin{array}{l}\text { disjoint sequences } \\
\text { of length } 36 \text { and weight } 25\end{array}$} & $\begin{array}{l}\text { NPAF } \\
n \geqslant 36\end{array}$ \\
\hline$(26,26,26,26)$ & disjoint & from Golay & $\begin{array}{l}\text { NPAF } \\
n \geqslant 26\end{array}$ \\
\hline$(14,14)$ & $\begin{array}{l}a b-b-b b a a \\
b-a a a-a b b\end{array}$ & $\begin{array}{c}-b a-b a-b-b b \\
a b a-b a-a-a\end{array}$ & $\begin{array}{c}\text { NPAF } \\
7 n\end{array}$ \\
\hline$(17,17)$ & $\begin{array}{l}\text { a }-\mathrm{a} \text { a } \text { a a a }-\mathrm{a} \text { a } 0 \\
\mathrm{c}-\mathrm{c} \text { c c c c }-\mathrm{c} \text { c } 0\end{array}$ & $\begin{array}{l}c-c-c c c c c-c-c \\
a-a-a\end{array}$ & $\begin{array}{c}\text { PAF } \\
9 n\end{array}$ \\
\hline
\end{tabular}

Table 2: (continued). 


\section{SOME GENERAL RESUltS}

We now consider the use of sequences with zero non-periodic autocorrelation function to make an amicable set of matrices. We refer the reader to $[6,8]$ for any undefined terms.

THEOREM 2. (General construction.) Let $X, Y$ be two disjoint $(0, \pm 1)$ sequences with zero non-periodic autocorrelation function of length $n$ and weight $k$, Let $a, b, c, d$ be commuting variables and write $a V, b W$ for the circulant (type 1) matrices of order $n$ formed by using the first rows with the elements of $X$ multiplied by $a$ and the elements of $Y$ multiplied by $b$ respectively.

Let $A_{i}$ be the circulant matrices of order $n$ given by

$$
A_{1}=a V+b W \quad A_{2}=c V+d W \quad A_{3}=d V-c W \quad A_{4}=b V-a W
$$

then $\left\{A_{i}\right\}_{i=1}^{4}$ is a short amicable set satisfying

$$
\sum_{i=1}^{2}\left(A_{2 i-1} A_{2 i}^{T}-A_{2 i} A_{2 i-1}^{T}\right)=0
$$

and the additive property

$$
\sum_{i=1}^{4}\left(A_{i} A_{i}^{T}\right)=k\left(a^{2}+b^{2}+c^{2}+d^{2}\right) I_{n} .
$$

Proof: Now $A_{1}=a V+b W$, where $V, W$ are disjoint $(0, \pm 1)$ circulant (type 1 or group developed also suffice) matrices of order $n$ which satisfy $V V^{T}+W W^{T}=k I_{n}$, and similarly for the other $A_{j}, j=2,3,4$.

Then

$$
A_{1} A_{1}^{T}=(a V+b W)\left(a V^{T}+b W^{T}\right)=a^{2} V V^{T}+b^{2} W W^{T}+a b\left(V W^{T}+W V^{T}\right) .
$$

Hence

$$
\begin{aligned}
\sum_{i=1}^{4}\left(A_{i} A_{i}^{T}\right) & =\left(a^{2}+b^{2}+c^{2}+d^{2}\right)\left(V V^{T}+W W^{T}\right) \\
& =k\left(a^{2}+b^{2}+c^{2}+d^{2}\right) I_{n},
\end{aligned}
$$

Now

$$
\begin{aligned}
A_{1} A_{2}^{T}-A_{2} A_{1}^{T} & =(a V+b W)\left(c V^{T}+d W^{T}\right)-(c V+d W)\left(a V^{T}+b W^{T}\right) \\
& =(a d-b c) V W^{T}+(-a d+b c) W V^{T}
\end{aligned}
$$

and

$$
\begin{aligned}
A_{3} A_{4}^{T}-A_{4} A_{3}^{T} & =(d V-c W)\left(b V^{T}-a W^{T}\right)-(b V-a W)\left(d V^{T}-c W^{T}\right) \\
& =(-a d+c b) V W^{T}+(a d-c b) W V^{T}
\end{aligned}
$$

Thus summing over the four $A_{i}$ we see they form a short amicable set satisfying the additive property. 


\begin{tabular}{|l|c|}
\hline Type & ZERO \\
\hline$(1,1,1,1)$ & NPAF $n \geqslant 1$ \\
$(2,2,2,2)$ & NPAF $n \geqslant 2$ \\
$(4,4,4,4)$ & NPAF $n \geqslant 4$ \\
$(5,5,5,5)$ & NPAF $n \geqslant 6$ \\
$(8,8,8,8)$ & NPAF $n \geqslant 8$ \\
$(10,10,10,10)$ & NPAF $n \geqslant 10$ \\
$(13,13,13,13)$ & NPAF $n \geqslant 18$ \\
$(16,16,16,16)$ & NPAF $n \geqslant 16$ \\
$(17,17,17,17)$ & NPAF $n \geqslant 26$ \\
$(20,20,20,20)$ & NPAF $n \geqslant 20$ \\
$(25,25,25,25)$ & NPAF $n \geqslant 36$ \\
$(26,26,26,26)$ & NPAF $n \geqslant 26$ \\
\hline
\end{tabular}

Table 3: Short amicable sets from Corollary 1

Corollary 1. Let $X, Y$ be a pair of disjoint $(0, \pm 1)$ sequences with zero nonperiodic autocorrelation function of length $n$ and weight $k$. Then there exists a short amicable set which can be used to form an $O D(4 n ; k, k, k, k)$.

Proof: Use the sequences as in the theorem to form an amicable set with the additive property. Then use this set in the Goethals-Seidel array to obtain the result. [

For $\alpha, \beta, \gamma, \delta, \varepsilon, \phi, \psi, \mu, \nu$ non-negative integers, Koukouvinos and Seberry [5, p.160] show that there exist two disjoint $(0, \pm 1)$ sequences, with zero non-periodic autocorrelation function, of length $\geqslant n, n \in N=\left\{2 \times 2^{\alpha} 6^{\beta} 10^{\gamma} 9^{\delta} 14^{\varepsilon} 18^{\phi} 26^{\psi} 24^{\mu} 34^{\nu}\right\}$ and weight $k, k \in K=\left\{2^{\alpha} 5^{\beta} 10^{\gamma} 13^{\delta} 17^{\varepsilon} 25^{\phi} 26^{\psi} 34^{\mu} 50^{\nu}\right\}$. These give the results presented in Table 3 .

\section{REFERENCES}

[1] S. Georgiou, C. Koukouvinos and J.Seberry, 'Short amicable sets', (submitted).

[2] A.V. Geramita and J. Seberry, Orthogonal designs: Quadratic forms and Hadamard matrices, Lecture Notes in Pure and Applied Mathematics 45 (Marcel Dekker, New York, 1979).

[3] W.H. Holzmann and H. Kharaghani, 'On the Plotkin arrays', Australas. J. Combin. 22 (2000), 287-299.

[4] H. Kharaghani, 'Arrays for orthogonal designs', J. Combin. Designs 8 (2000), 166-173.

[5] C. Koukouvinos and J. Seberry, 'New weighing matrices and orthogonal designs constructed using two sequences with zero autocorrelation function - a review', J. Statist. Plann. Inference 81 (1999), 153-182.

[6] J. Seberry and R. Craigen, 'Orthogonal designs', in CRC Handbook of combinatorial designs, (C.J. Colbourn and J.H. Dinitz, Editors) (CRC Press, Boca Raton, 1996), pp. 400-406. 
[7] J. Seberry and A.L. Whiteman, 'New Hadamard matrices and conference matrices obtained via Mathon's construction', Graphs Combin. 4 (1988), 355-377.

[8] J. Seberry and M. Yamada, 'Hadamard matrices, sequences and block designs', in Contemporary design theory: A collection of surveys, (J.H. Dinitz and D.R. Stinson, Editors) (J. Wiley and Sons, New York, 1992), pp. 431-560.

Department of Mathematics

National Technical University of Athens

Zografou 15773

Athens

Greece
School of IT and Computer Science

University of Wollongong

Wollongong NSW 2522

Australia 